\title{
Comunicación
}

\section{Descripción Osteológica del Puma Andino (Puma concolor): I. Esqueleto Apendicular}

\author{
Bone Description of the Andean Puma (Puma concolor): I. Appendicular skeleton \\ Joel I. Pacheco ${ }^{1,3}$, Celso Zapata ${ }^{2}$
}

\section{RESUMIEN}

Se realizó el estudio del esqueleto apendicular del puma andino (Puma concolor) en dos especímenes adultos: un macho y una hembra, decomisados en Cusco, Perú, por el Servicio Nacional Forestal y de Fauna Silvestre (SERFOR). La obtención de las piezas óseas se realizó siguiendo la técnica estándar de disección osteológica. La descripción de los términos anatómicos se hizo según la Nómina Anatómica Veterinaria. Las características más resaltantes del esqueleto apendicular fueron descritas. En general, las eminencias de los huesos no son tan notorias y solo se presentan como zonas rugosas; el radio y cúbito completos y con carillas articulares bien definidas. A nivel de las manos sobresale el carpo intermedio radial, los metacarpos ligeramente cilíndricos y curvados en la superficie palmar, la tercera falange en caso del primer dedo está bastante desarrollada y con una prominente apófisis ungeal, característico de estas especies. Se presenta una cabeza de fémur esférica y a nivel de los cóndilos, dos sesamoideos del gastrocnemio bien definidos; tibias y peronés completos. En los tarsos, en número de siete, sobresalen el astrágalo y el calcáneo. Los metatarsianos y falanges son muy similares a los de la mano. A nivel del coxal, los huesos compactos se asemejan a los del gato doméstico.

Palabras clave: Puma concolor; esqueleto apendicular; anatomía; osteología

\section{Abstract}

The appendicular skeleton of the Andean puma (Puma concolor) of two specimens (one male, one female) is described. Both animals were seized in Cusco, Peru by the National Forest and Wildlife Service (SERFOR). The bones were obtained following the standard technique of osteological dissection. The description of the anatomical terms

\footnotetext{
${ }^{1}$ Grupo de Investigación en Fauna Silvestre, Estación Experimental del Centro de Investigación IVITA, sede Maranganí, Universidad Nacional Mayor de San Marcos, Cusco, Perú

${ }^{2}$ Escuela de Medicina Veterinaria, Universidad Nacional San Antonio Abad del Curso, Perú

${ }^{3}$ E-mail:jpachecoc@unmsm.edu.pe
}

Recibido: 9 de enero de 2017

Aceptado para publicación: 18 de mayo de 2017 
was made according to the Veterinary Anatomical List. The most outstanding characteristics of the appendicular skeleton were described. In general, the eminences of the bones are not so conspicuous and only appear as rough areas. Complete radius and ulna with well-defined articular veneers. At the level of the hands the radial carpi stand out, the metacarpi slightly cylindrical and curved in the palmar surface, the third phalanx of the first finger is quite developed and with the prominent ungueal apophysis characteristic of these species. A well-defined spherical femoral head is present at the level of the two well-defined sesamoid gastrocnemius condyles; tibia and fibula are complete. In the tarsus, in number of seven, excel the astragalus and the calcaneus. The metatarsals and phalanges are very like those of the hand. At the level of the hip, the compact bones resemble those of the domestic cat.

Key words: Puma concolor; appendicular skeleton; anatomy; osteology

\section{INTRODUCCIÓN}

El puma andino (Puma concolor) está distribuido desde Canadá hasta el sur de Chile, ubicándose en la costa, valles interandinos y la Amazonía (OSINFOR 2011), describiéndose incluso a $5800 \mathrm{msnm}$ (Clavijo y Ramírez, 2009). Es de tamaño grande, esbelto y musculoso, pelaje corto y uniforme, de color marrón leonado, con la región ventral más pálida que el dorso, cola larga del color del dorso y con el extremo de color negruzco (OSINFOR, 2011).

El hábitat del puma andino se ha reducido a zonas muy apartadas (Zeballos et al., 2001). El puma es considerado por los pobladores rurales como uno de los animales más perjudiciales por la cantidad de animales que depredan (Deusta et al., 2008). Asimismo, el gobierno peruano, según D.S. 034-2004-AG del 17 de septiembre de 2004 (Minagri, 2004), lo considera como una especie casi amenazada.

Los estudios anatómicos para esta especie son limitados e incluyen la descripción de los nervios del antebrazo y mano (Sánchez et al., 2013), la discriminación del aparato masticatorio en pumas juveniles y adultos (Biknevicius 1996) y la morfología craneal (Sims, 2012). Una importante limitación se debe a la restricción de acceso a los especímenes. El presente estudio hace una descripción del esqueleto apendicular del Puma concolor.

\section{Materiales y MéTOdos}

El estudio se hizo con dos pumas andinos adultos. Un macho de $52 \mathrm{~kg}$ de peso y una hembra de $45 \mathrm{~kg}$ de peso, que fueran decomisados por caza ilegal en las provincias de Canchis y Canas, respectivamente, del departamento de Cusco (Perú), y remitidos al laboratorio de fauna silvestre de la estación experimental del Centro de Investigación IVITA de la Universidad Nacional Mayor de San Marcos, en Maranganí, Cusco. El decomiso y envío al laboratorio se hizo por el Servicio Nacional Forestal y de Fauna Silvestre (SERFOR), mediante acta de custodia temporal N. ${ }^{\circ}$ 01-2015-SERFOR-ATFFS-Cusco.

Para obtener el esqueleto se procedió con la técnica descrita por Cañete et al. (2014). En la descripción del esqueleto apendicular se siguieron las pautas descritas por Getty et al. (1990) y Dyce et al. (2007). Los términos utilizados están de acuerdo a la Nómina Anatómica Veterinaria (Schaller, 1996). El procedimiento para medir las piezas óseas se hizo según von den Driesch 
(1976). Para la medición de las piezas óseas se utilizó un calibrador vernier (Dial caliper ${ }^{\circledR}$, EEUU) de 150 x $0.02 \mathrm{~mm}$ y una regla milimetrada metálica (Matrix $\left.{ }^{\circledR}\right)$.

\section{Resultados}

El tamaño de las piezas óseas del esqueleto apendicular, expresadas en centímetros se presenta en el Cuadro 1.

\section{Miembro anterior (Figura 1)}

Escápula. Es relativamente estrecha. El ángulo craneal no está bien definido, siendo ligeramente redondeado, continuándose con el borde craneal, el cual es convexo. La tuberosidad supraglenoidea es poco prominente, la apófisis coracoides bastante ganchosa, el cuello no bien definido, presentando una pequeña tuberosidad en la parte caudal. La tuberosidad de la espina poco prominente, la apófisis hamata dirigida ventralmente y a nivel de la cavidad glenoidea.

Húmero. El cuerpo del húmero es aplanado lateralmente en su tercio proximal y torcido sobre su eje hacia distal. La tuberosidad deltoidea se presenta como una rugosidad; el tubérculo mayor no está dividido, el tubérculo menor es pequeño, y el surco intertuberal no dividido y dirigido medialmente. En la extremidad distal, la tróclea está bien excavada y con cóndilos prominentes. El agujero supracondilar bien definido y los epicóndilos bastante prominentes.

Cubito. En general, proximalmente más grueso y distalmente delgado, así como aplanado lateralmente, presentando un surco en ambos lados. La apófisis olecraniana es poco prominente y presenta un pequeño surco. Distalmente presenta una apófisis estiloides prominente.

Radio. Hueso largo, estrecho proximalmente y grueso distalmente, aplanado lateralmente. La cara medial convexa y la cara lateral cón-
Cuadro 1. Tamaño $(\mathrm{cm})$ de las piezas óseas $^{1}$ del esqueleto apendicular del puma andino (Puma concolor)

\begin{tabular}{|c|c|c|c|}
\hline & & $\begin{array}{l}\text { Macho } \\
(n=1)\end{array}$ & $\begin{array}{l}\text { Hembra } \\
(\mathrm{n}=1)\end{array}$ \\
\hline Pelvis & & 20.5 & 18.4 \\
\hline Fémur & & 26.0 & 23.4 \\
\hline Tibia & & 23.7 & 21.4 \\
\hline Peroné & & 19.7 & 19.7 \\
\hline \multirow[t]{4}{*}{ Falange 1} & Dedo 2 & 3.1 & 3.0 \\
\hline & Dedo 3 & 3.7 & 3.7 \\
\hline & Dedo 4 & 3.6 & 3.4 \\
\hline & Dedo 5 & 3.2 & 3.2 \\
\hline \multirow[t]{4}{*}{ Falange 2} & Dedo 2 & 2.1 & 2.| \\
\hline & Dedo 3 & 2.7 & 2.7 \\
\hline & Dedo 4 & 2.5 & 2.5 \\
\hline & Dedo 5 & 3.2 & 2.2 \\
\hline \multirow[t]{4}{*}{ Falange 3} & Dedo 2 & 1.8 & 1.7 \\
\hline & Dedo 3 & 2.0 & 2.2 \\
\hline & Dedo 4 & 2.1 & 2.0 \\
\hline & Dedo 5 & 2.0 & \\
\hline Escápula $^{2}$ & & 15.4 & 14.9 \\
\hline Húmero & & 22.5 & 20.2 \\
\hline Radio & & 18.1 & 16.1 \\
\hline Cúbito & & 22.4 & 20.2 \\
\hline \multirow[t]{5}{*}{ Falange 1} & Dedo 1 & 2.5 & 2.5 \\
\hline & Dedo 2 & 2.0 & 3.3 \\
\hline & Dedo 3 & 3.7 & 3.4 \\
\hline & Dedo 4 & 3.0 & 3.0 \\
\hline & Dedo 5 & 2.7 & 3.0 \\
\hline \multirow[t]{4}{*}{ Falange 2} & Dedo 2 & 2.2 & 2.8 \\
\hline & Dedo 3 & 2.5 & 2.5 \\
\hline & Dedo 4 & 2.5 & 2.5 \\
\hline & Dedo 5 & 2.1 & 2.1 \\
\hline \multirow{5}{*}{ Falange 3} & Dedo 1 & 3.5 & 3.1 \\
\hline & Dedo 2 & 2.9 & 2.6 \\
\hline & Dedo 3 & 2.6 & 2.6 \\
\hline & Dedo 4 & 2.1 & 2.3 \\
\hline & Dedo 5 & 2.3 & 2.1 \\
\hline
\end{tabular}

${ }^{1}$ Longitud mayor

${ }^{2}$ Longitud mayor en relación a la espina escapular

cava. La extremidad proximal muestra un cuello bien definido, y la extremidad distal presenta surcos para los tendones de los extensores y una apófisis estiloides bien definida. 


\section{Mano}

- Los carpos comprenden siete huesos tres en la fila proximal y cuatro en la fila distal - sobresaliendo el carpo intermediorradial.

- Los metacarpianos son huesos largos, cilíndricos y ligeramente curvos. El primer metacarpiano es el más corto, el tercer y cuarto metacarpiano son los más largos en comparación con el segundo y el quinto.

- Las falanges son en número de tres para los dedos 2, 3, 4, 5, y en número de dos para el dedo 1. En la tercera falange se forman las garras: en los dedos 2, 3, 4 y 5 mantienen su tamaño. En el primer dedo, el tamaño de la tercera falange es aproximadamente dos veces el tamaño con relación a los otros dedos. Presentan en la base una superficie articular amplia para la segunda falange con un gran tubérculo flexor y a cada lado un foramen. Dorsal a la superficie articular existe una eminencia para los extensores.

\section{Miembro Posterior (Figura 2)}

Coxal. Formado por tres huesos:

- $\quad$ Ilion. Ligeramente paralelo entre el ilion y el plano medio. La superficie glútea cóncava poco excavada, la superficie sacropelviana bien definida y paralela presentando la espina iliaca dorsal craneal y caudal, la espina iliaca ventral craneal y caudal, y el cuerpo comprimido lateralmente presentando una buena eminencia iliopúbica.

- Isquion. Aplanado. La tuberosidad isquiática gruesa, espina isquiática poco desarrollada, el arco isquiático menor poco profundo, el ángulo caudal medial poco diferenciado.

- Pubis. El borde medial pronunciado ventralmente.

Acetábulo. Denota una forma circular interrumpida por una profunda escotadura acetabular. La superficie articular no está dividida y la fosa acetabular es amplia.
Agujero obturador. Denota una forma elíptica.

Fémur. Hueso largo, recto y cilíndrico. La extremidad proximal presenta una cabeza esférica, el cuello bien definido y la fóvea poco profunda. El trocánter mayor no sobrepasa la altura de la cabeza del fémur, el cual está dividido en dos porciones: craneal y caudal. La fosa trocantérica profunda, la cresta intertrocantérica ligeramente oblicua, trocánter menor pequeño y pronunciado. En la extremidad distal, la tróclea es sagital. La superficie dorsal de los cóndilos presenta una superficie articular para los sesamoideos del gastrocnemio. La fosa intercondílea amplia y profunda. Las tuberosidades supracondíleas aparecen como rugosidades.

Rótula. Presenta dos caras, dos bordes una base y un vértice.

Sesamoideos del gastrocnemio. Cada fémur presenta dos huesos sesamoideos - uno medial y otro lateral -, ubicados en la cara dorsal de los cóndilos del fémur. El lateral es más grande y curvo en relación con el sesamoideo medial.

Tibia. Tiene casi la misma longitud que el fémur. La forma prismática se proyecta distalmente. La tuberosidad y la cresta son poco pronunciados, la fosa de la tibia un poco profunda, y en la extremidad proximal presenta la superficie articular para el peroné. En la extremidad distal, los surcos de la cóclea son oblícuos; a nivel del surco lateral, uno de sus lados esta reemplazado por el peroné con el que se articula. El agujero interóseo se extiende por toda la longitud del hueso.

Peroné. Hueso completo y delgado. La extremidad proximal presenta superficie articular para con la tibia, y presenta una apófisis de dirección craneal. El cuerpo es delgado y recto en el tercio proximal y medio con tres superficies y en el tercio distal solo dos superficies. La extremidad distal presenta una superficie articular para con la tibia, y presenta una eminencia de dirección lateral. 


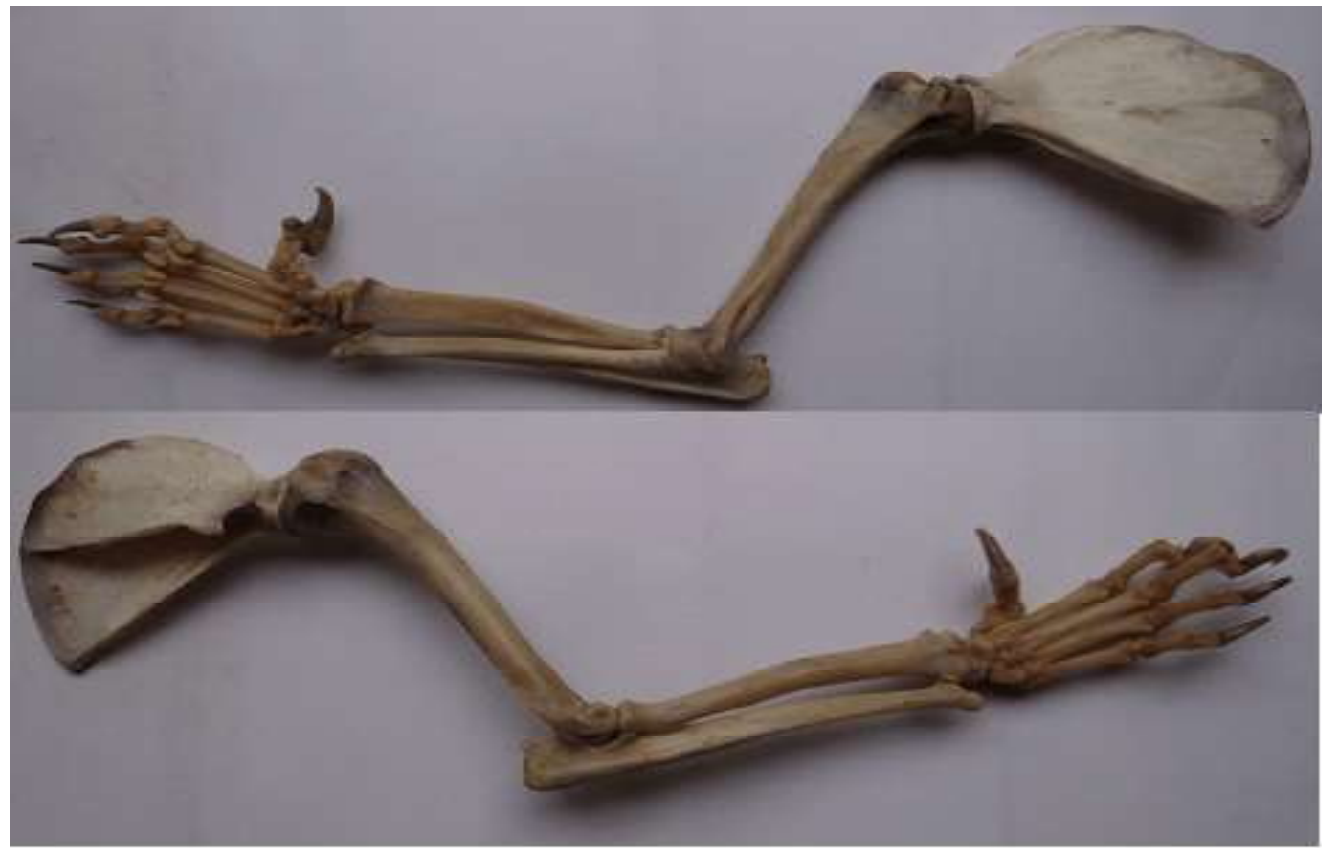

Figura 1. Miembro anterior del puma andino (Puma concolor). Foto superior: escápula, húmero, radio, cúbito (vista medial), mano (vista palmar). Foto inferior: escápula, húmero, radio, cúbito (vista lateral), mano (vista dorsal)

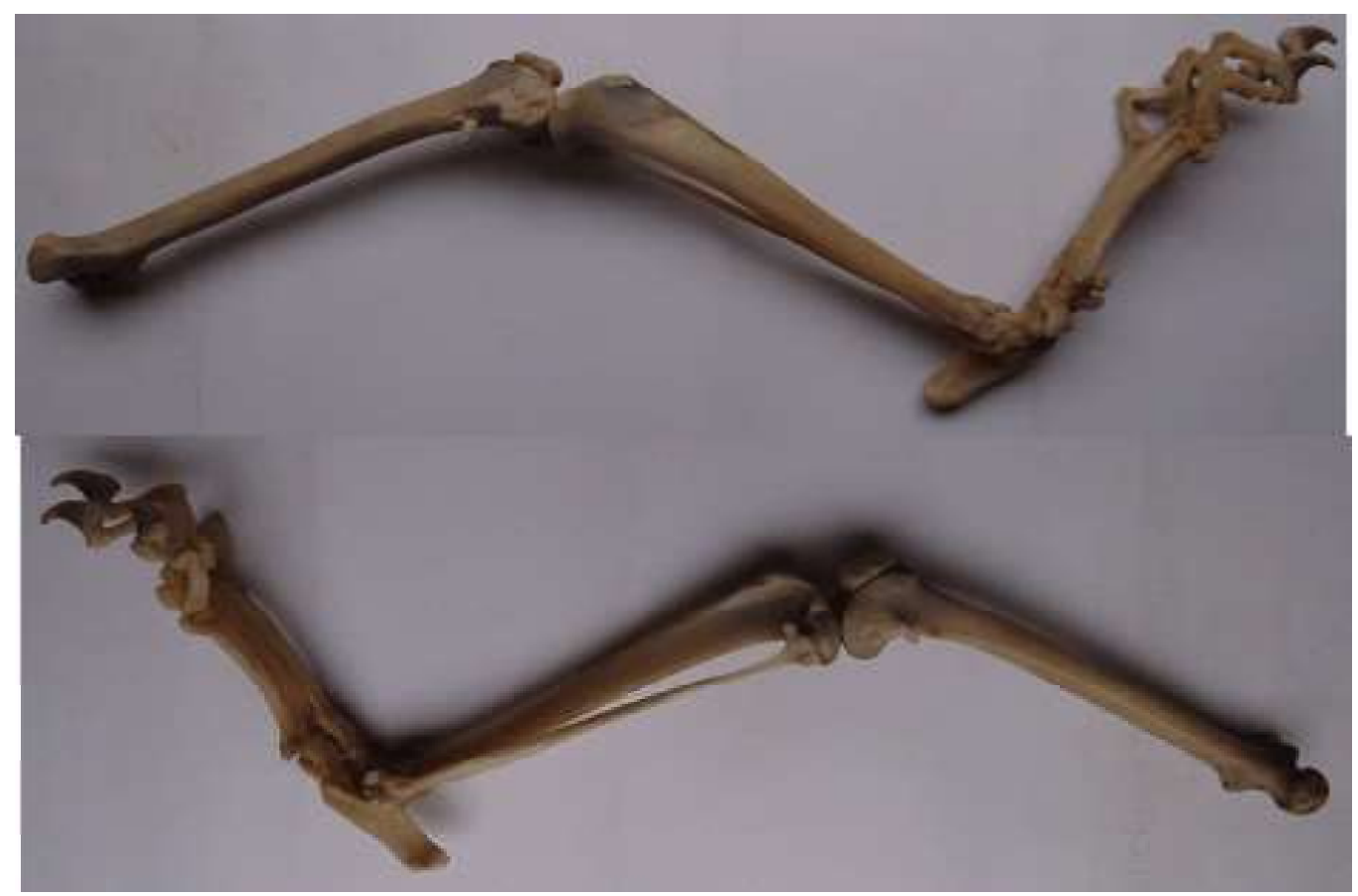

Figura 2. Miembro posterior del puma andino (Puma concolor). Foto superior: Vista medial del fémur, tibia (posición medial), peroné (posición lateral), tarso, metatarsos y falanges. Foto inferior: Vista lateral del fémur, tibia (posición medial), peroné (posición lateral), tarso, metatarsos y falanges 


\section{Pie}

- Los tarsos comprenden siete huesos.

- El astrágalo (tarso tibial) presenta un cuerpo, cuello y cabeza. La tróclea es ligeramente oblicua, siendo la cresta lateral más prominente que la medial. La cabeza está medial y presenta una superficie articular amplia y convexa para articularse con el tarso central. El astrágalo presenta tres superficies articulares para con el calcáneo.

- El calcáneo (tarso peroneo) presenta la tuberosidad calcánea con un surco sagital bien excavado. Tanto la apófisis coracoides como el sutentáculo son algo cortos. La superficie articular para el IV hueso del tarso es triangular y ligeramente cóncava y hacia lateral presenta un surco bien marcado.

- El central del tarso es irregularmente cuadrilátero. La superficie articular proximal es ligeramente cóncava y en su borde plantar presenta dos tubérculos plantares separados por un surco donde el tubérculo medial es más prominente.

- Se encuentra superficies articulares para el calcáneo, I, II, III, IV tarsales. El I y II tarsianos son los más pequeños de la fila distal. El III tarsiano es un hueso irregular alargado de dorsal a plantar. El IV tarsiano es el más alto de la fila distal. Las superficies articulares, tanto proximales como distales, son amplias y ligeramente planas. En la superficie lateral presenta un surco oblicuo bien excavado.

- Metatarso: presenta cinco huesos metatarsianos, de los cuales el primero es vestigial. En los otros cuatro, sobresale el tercer metatarsiano. En general, los metatarsianos presentan una diáfisis cilíndrica donde la superficie dorsal es convexa y la plantar es cóncava. En la extremidad distal, en su superficie plantar, existe la presencia de dos huesos sesamoideos para cada metatarsiano.

- Las falanges también son en número de tres para los dedos 2, 3, 4, 5. Muy similares a los de la mano.

\section{Discusión}

Los huesos descritos del esqueleto apendicular del puma presentan características óseas que también son encontradas en el gato doméstico, tal como lo describen Getty et al. (1990) y Dyce et al. (2007). De igual forma, estos relieves óseos han sido enunciados en el trabajo de Concha et al. (2004), donde al describir la musculatura del miembro torácico del puma, dichos relieves son indicados como puntos de origen e inserción de músculos, dando evidencia de su presencia.

Las características encontradas en el metacarpiano se asemejan con lo descrito por Morales-Mejía et al. (2010), donde indica que los metacarpianos son curvos y que el tercer metacarpiano es ancho y curvo a diferencia de los otros metacarpianos.

Los rasgos anatómicos encontrados a nivel de las falanges se asemejan a lo descrito por Morales-Mejía y Arroyo-Cabrales (2012), quienes indican que la presencia de falanges cortas con superficies prominentes para la inserción de tendones y apófisis ungelar con una garra curva es una característica que corresponde a la familia Felidae y que dicha curvatura que presentan las falanges estaría directamente relacionada con la retracción de la garra.

A nivel de los huesos del tarso, los huesos más descritos han sido el astrágalo y el calcáneo, y muchas de sus características concuerdan con lo descrito por Morales-Mejía y Arroyo-Cabrales (2012), quienes señalan que el astrágalo, el tener superficies articulares anchas y al presentar una apófisis calcánea recta con surco profundo y una superficie articular para el cuboide, son características que corresponden a la familia Felidae. Asimismo, Morales-Mejía et al. (2010) indica que el hecho de presentar una carilla articular para el cuboide en forma triangular, y el tener una prolongación en el borde lateral, son características que sirven para diferenciarlos de otros grandes felinos. 
A nivel de los metatarsianos, los datos de este estudio comparen lo descrito por Morales-Mejía y Arroyo-Cabrales (2012), donde la curvatura de la diáfisis es una característica de la familia Felidae, y con Morales-Mejía et al. (2010) que indica que el primer metatarsiano es reducido y los demás son curvos.

La ubicación topográfica de los sesamoideos del gastrocnemio en el miembro pelviano se asemeja a la del gato doméstico, tal como lo describen Getty et al. (1990) y Dyce et al. (2007), resaltando el sesamoideo lateral con respecto al medial. Asimismo, la rótula se ubica casi vertical, presentando una base proximal y un ápice redondeado distal (Cerveny y Páral, 1995).

\section{Conclusiones}

- La tercera falange del dedo 1 es dos veces más grande que las otras terceras falanges.

- El esqueleto apendicular presenta muchas similitudes a la del gato doméstico.

\section{Agradecimientos}

Se agradece la colaboración del Ing. Francisco Franco (IVITA MaranganíUNMSM), de los biólogos Rubén Concha y Gisella Zans (SERFOR, Cusco) y del estudiante Jose Carlos Arias (UNSAAC).

\section{Literatura Citada}

1. Biknevicius A. 1996. Functional discrimination in the masticatory apparatus of juvenile and adult cougars (Puma concolor) and spotted hyenas (Crocuta crocuta). Can J Zool 74: 19341942. doi: 10.1139/z96-218

2. Cañete G, Sánchez J, Noda L. 2014. Ensamblaje artesanal de un esqueleto canino mediante variantes de la osteotecnia. REDVET 15(9). [Internet]. Dispo- nible en: www.veterinaria.org/revistas/ redvet/n090914./091410.pdf

3. Cerveny C, Paral V. 1995. Sesamoid bones of the knee joint of the Puma concolor. Acta Vet Brno 64: 79-82. doi: 10.2754/avb199564010079

4. Clavijo A, Ramírez G. 2009. Taxonomía, distribución y estado de conservación de los felinos suramericanos: revisión monográfica. Bol Cient Mus Hist Nat 13: 43-60.

5. Concha I, Adaro L, Borrony C, Altamirano C. 2004. Consideraciones anatómicas sobre la musculatura intrínseca del miembro torácico del puma (Puma concolor). Int J Morphol 22: 121125. doi: 10.4067/S0717-95022004000200004

6. Deustua I, Williams M, Vásquez P. 2008. Relaciones entre los pobladores rurales y los carnívoros altoandinos del distrito de Anco, Centro-Sur del Perú. Ecol Apl 7: 43-48. doi: 10.21704/rea.v7i12.358

7. Dyce KM, Sack WO, Wensing CJG. 2007. Anatomía veterinaria. $3^{\text {ra }}$ ed. México: Ed Manual Moderno. 920 p.

8. Getty R, Sisson S, Grosman J. 1990. Anatomía de los animales domésticos. $5^{\circ}$ ed. Barcelona: Salvat. $2302 \mathrm{p}$.

9. [MINAGRI] Ministerio de Agricultura. 2004. Decreto Supremo N. ${ }^{\circ} 034-$ 2004- AG. Normas legales. Diario el Peruano. [Internet]. Disponible en: http:/ /www.serfor.gob.pe/wp-content/ uploads/2016/03/ds04-2014-minagri.pdf

10. Morales-Mejia F, Arroyo-Cabrales J. Polaco O. 2010. Estudio comparativo de algunos elementos de las extremidades anteriores y posteriores y piezas dentales de puma (Puma concolor) y jaguar (Panthera onca). TIP Rev Esp Cienc Quím Biol 13: 73-90.

11. Morales-Mejia F, Arroyo-Cabrales J. 2012. Estudio comparativo de algunos elementos de las extremidades de las familias Felidae y Canidae (Mammalia, Carnívora). TIP Rev Esp Cienc Quím Biol 15: 75-84. 
12. [OSINFOR] Organismo de Supervisión de los Recursos Forestales y de Fauna Silvestre. 2011. Resolución Presidencial N. ${ }^{\circ}$ 007-2011-OSINFOR. Fichas técnicas de los mamíferos representativos de la amazonía Peruana. Disponible en: http://osinfor.gob.pe/portal/data/recurso/rp_007-2011-osinfor_aprueba_fichas_tecnicas_mamiferos. pdf

13. Sánchez HL, Silva LB, Rafasquino ME, Mateo AG, Zuccolilli GO, Portiansky EL, Alonso CR. 2013. Anatomical study of the forearm and hand nerves of the domestic cat (Felis catus), puma (Puma concolor) and jaguar (Panthera onca). Anat Histol Embryol 42: 99-104. doi: 10.1111/j.14390264.2012.01170.x
14. Schaller O. 1996. Nomenclatura anatómica veterinaria ilustrada. Zaragoza, España: Acribia. 622 p.

15. Sims M. 2012. Cranial morphology five felids: Acinonyx jubatus, Panthera onca, Panthera pardus, Puma concolor, Uncia uncia. Russian J Theriol 11: 157-170. doi: 10.15298/ rusjtheriol.11.2.05

16. von den Driesch A. 1976. A guide to the measurement of animal bones fron archaeological sites. Massachusetts, USA: Harvard University. 136 p.

17. Zeballos H, Pacheco V, Baraybar L. 2001. Diversidad y conservación de los mamíferos de Arequipa. Rev Peru Biol 8: 94-104. doi: 10.15381/rpb.v8i2.6564 